\title{
Review of Dicompass DICOM Camera App for Android
}

\author{
Dawn Cram $^{1} \cdot$ Richard Stratton $^{1} \cdot$ Shawn Clark $^{1}$
}

Published online: 14 December 2015

(C) Society for Imaging Informatics in Medicine 2015

\section{App Specs}

Seller: Medoro s.r.o.

Category: Medical

Updated: October 02, 2015

Version: 1.2 .5

Size: $3.2 \mathrm{M}$

Rating: Rated 4+ Family sharing: N/A

Compatibility: Requires Android 4.0.3 and up

Languages: English

Czech

German

Russian

Polish

Spanish

French

Slovak

App icon URL: https://play.google.com/store/apps/details? id $=$ org.medoro.dicompasscamera

App name: Dicompass DICOM Camera

App developer: MEDORO s.r.o.

Dawn Cram

dcram@med.miami.edu

Richard Stratton

rsstratton@med.miami.edu

Shawn Clark

s.clark11@med.miami.edu

1 Information Technology-Enterprise Imaging, University of Miami, Miami, FL, USA
App developer Website: http://www.dicompass.cz/

App price: Free for evaluation. US\$8 for 1 month; US\$82 for 1 year license; and US\$160 for 3 years. Contact company for site licensing options.

Apple App Store URL: N/A

Google Play Store URL: https://play.google.com/store/apps/ details?id=org.medoro.dicompasscamera

Category: clinical, medical

Tags: clinical DICOM, MWL, XC, clinical photo, wireless, Android camera

Works offline: Yes

FDA approval: N/A

Promotion code: JDI-DICOMPASS (10 \% discount for directly purchased licenses.)

\section{Quick Review}

(1 star: lowest/5 stars: highest)

Overall rating (1-5): 5

Content (1-5): 5

Usability (1-5): 4

Pros Configurable settings provide the flexibility to support various clinical photography workflows and integrations across different organizations and specialties.

Cons Only available for Android devices

At a Glance Android camera app, offering clinicians an intuitive method to associate patient and anatomical information to clinical photographs utilizing DICOM. 


\section{Full Review}

\section{Introduction}

Clinical photos are frequently acquired by multiple specialties to document and diagnose medical conditions. This app answers the challenge of providing a standard-based, wireless method for acquiring and storing clinical photos to a central archive with associated patient and image-specific metadata.

\section{Purpose/Features/Content}

The purpose of this app is to acquire and wirelessly transmit clinical photos with associated patient and image-specific information, utilizing DICOM standards.

The app supports multiple methods to enter patient demographics including DICOM MWL, barcode scanning, PID query, and manual entry (Fig. 1).

Once the patient information is selected, or manually entered, a device-configurable localization wizard launches allowing the clinician to select the body part, anatomical position, and disorder being imaged. The localization selections build the series and/or study description sent with the image metadata. The localization presets are also device configurable. Separate SUIDs can be generated for each body part selected and without reselecting or reentering the patient; this feature is also device configurable. Photos are acquired and transmitted as DICOM over a wireless network (Fig. 2).

If a wi-fi connection is not immediately available, the images will remain in queue until wi-fi is reestablished.

Both JPEG and uncompressed image format (little endian) are supported. The app also supports various resolutions including HD (720p), full HD (1080p), 4K $(2160 \mathrm{p})$ or the camera's original resolution and provides an image cropping tool.

Multiple security features are available including deletion of cached images and database record upon successful send, randomly generated file names, and LDAP support (Fig. 3). Devices can effectively run mobile device management software, in conjunction with the app, to provide an additional layer of security and kiosk-mode support.

Many settings and preset selections are device configurable, providing flexibility for varied service lines taking clinical photos. The service lines benefitting from the app may include dermatology, plastics, ophthalmology, otolaryngology, emergency services, and wound care.

\section{Usability}

The app offers an intuitive, easy-to-use interface for clinicians. It is sophisticated enough to provide granular data for the clinician when condition tracking is desired. Anyone with basic smartphone experience, of any platform, will find the touch screen interface familiar and easy to learn.

\section{Good}

The app provides flexibility to support varied organizational workflows and specialty-specific configurations. The app's conformance with DICOM standards proves invaluable in enabling image-associated information to be passed to an image archive.

The camera driver is very stable and acquires high-quality images.
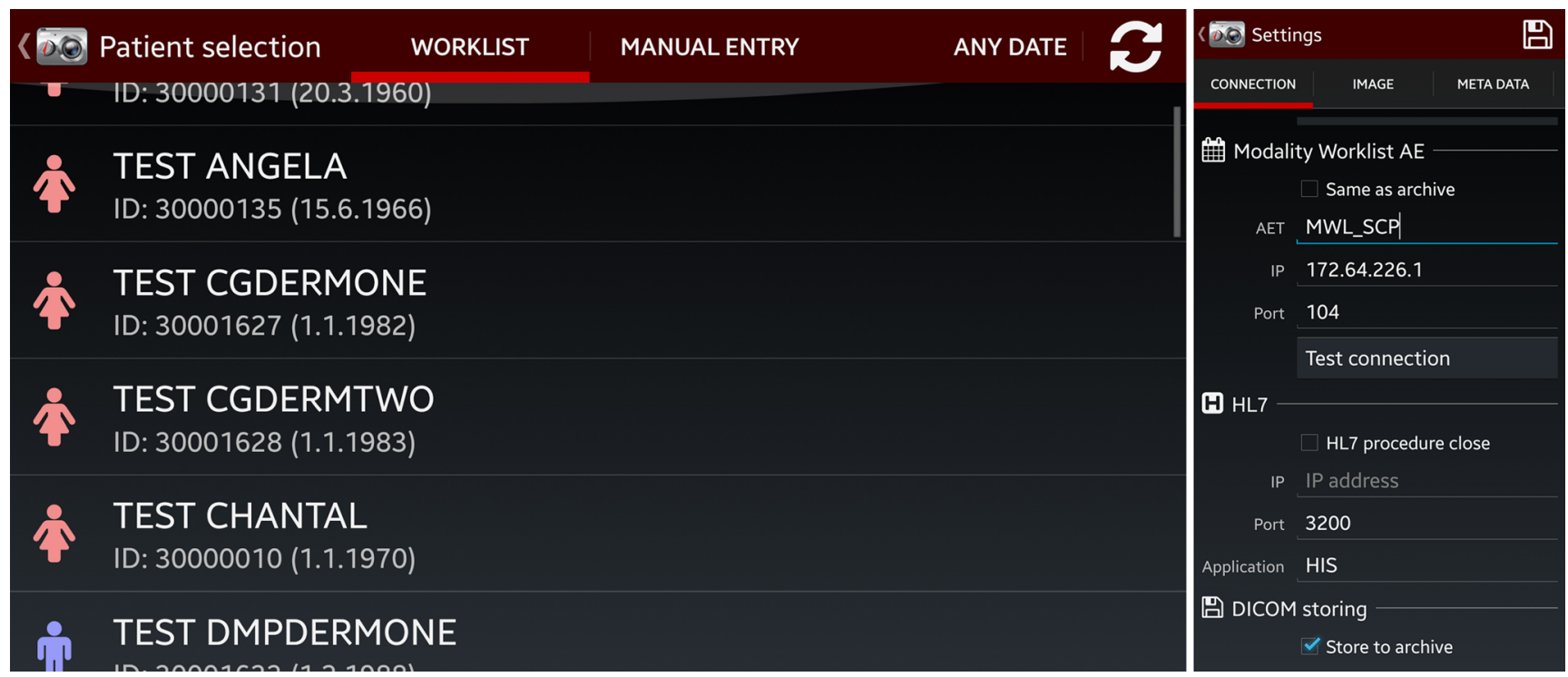

Fig. 1 Varied methods to identify a patient are available including DICOM modality worklist or manual entry 
Fig. 2 The touch screen body mapping is easy to use and can include an extensive array of configurable options to identify specific body part, laterality, anatomical position, and disorders

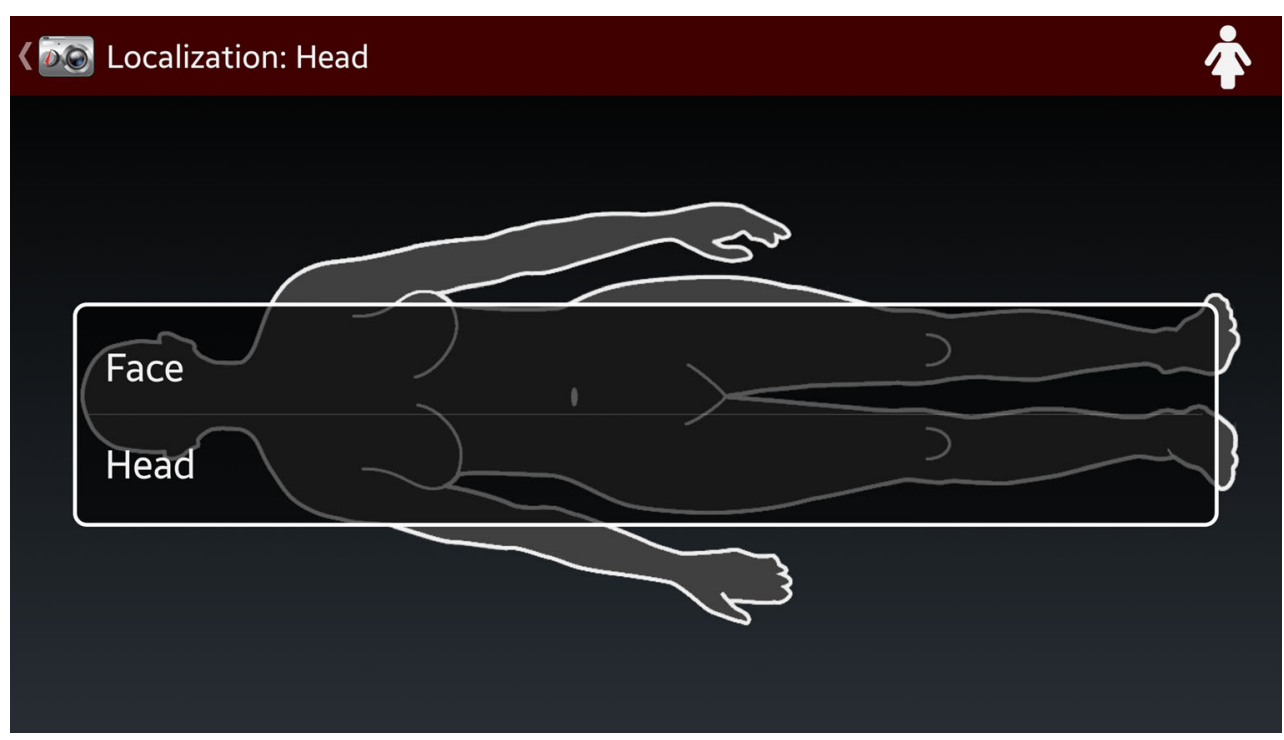

Fig. 3 Administrative security settings can be password protected and include options for LDAP integration, randomized file names, cache purging, and other features 


\section{Room for Improvement}

When a macro lens is used, lighting conditions are not optimal with built-in flash or if the auto focus has difficulty, and working around the limits of the driver can be challenging. The addition of an advanced operations mode that includes functions such as touch screen focusing, shutterpriority (or a setting to lock the shutter speed at $1 / 125$ or higher to prevent camera shake during macro capture), exposure compensation, or access to the camera's inherent scene presets may improve user experience and capture with a macro lens on Android interchangeable lens cameras. 